\title{
ON COMPLETELY DECOMPOSABLE GROUPS
}

JOHN S. P. WANG

In this note I shall give some theorems concerning completely decomposable groups. I use the same definition and follow the same notations as those in the book [1].

Definition. A subgroup $H$ of a torsion free group $G$ is said to be regular if the elements of $H$ have the same types in $H$ and $G$.

LEMMA 1. If $H$ is a regular subgroup of a torsion free group $G, G \geqq K$ $\geqq H$ and $K / H$ is torsion, then $K$ is also a regular subgroup of $G$.

Proof. For any $k \in K$, we have $T_{K}(k)$ (type of $k$ in $K$ ) $\leqq T_{G}(k)$. Since $K / H$ is torsion, there is an integer $n \neq 0$ such that $n k \in H$. From the fact that $H$ is regular, we have $T_{G}(k)=T_{G}(n k)=T_{H}(n k) \leqq T_{K}(n k)$ $=T_{K}(k) \leqq T_{G}(k)$. Hence we get $T_{K}(k)=T_{G}(k)$.

From this lemma we see that any subgroup $K$ of a torsion free group with $G / K$ of bounded order is a regular subgroup of $G$.

THEOREM 1. $G=\sum_{\lambda \in \Lambda} G_{\lambda}$ (direct sum) where $\Lambda$ is a well ordered set with least element $0, G_{\lambda}$ has rank 1 for $\lambda>0$ and $T(x) \geqq T(y)$, whenever $x \in G_{\lambda}, y \in G_{\mu}$ with $\lambda<\mu$. Then for any regular subgroup $H$ of $G$ we have $H=\left(H \cap G_{0}\right) \oplus \sum_{\lambda>0} H_{\lambda}$ where $H_{\lambda} \cong G_{\lambda}$ or $H_{\lambda}=0$.

Proof. Define $G^{a}=\sum_{\lambda<a} G_{\lambda}$ and $H^{a}=H \cap G^{a}$. Then $H^{a+1} / H^{a}$ $=H^{a+1} /\left(H^{a+1} \cap G^{a}\right) \cong\left\{G^{a}, H^{a+1}\right\} / G^{a} \leqq G^{a+1} / G^{a} \cong G_{a}$. It is clear that $H^{a}$ is pure in $H$ for each $a \in \Lambda$. If $H^{a+1} \neq H^{a}$ and $a>0$, for any $x \in H^{a+1}$ and $x \notin H^{a}$ we have $T_{H}(x)=T_{G}(x)=T\left(G_{a}\right)$. Hence $T\left(G_{a}\right) \leqq T\left(H^{a+1} / H^{a}\right)$ $\leqq T\left(G_{o}\right)=T(x)$ for $a>0$. By

BAER's LEMMA. Let $S$ be a pure subgroup of the torsion free group $G$ such that (1) $G / S$ is of rank 1 ; (2) $G / S$ is of type a and (3) every element of $G$ not in $S$ is again of type a. Then $S$ is a direct summand of $G$.

[1, Lemma 46.3 , p. 163], $H^{a}$ is a direct summand of $H^{a+1}$ and $H^{a+1}=H^{a} \oplus H_{a}$, where $H_{a}$ either vanishes or is $\cong G_{a}$ for $a>0$ and $H_{0}=H \cap G_{0}$. With a simple transfinite induction we are led to the result $H^{a+1}$ is the direct sum of all $H_{\lambda}$ with $\lambda \leqq a$, and $H=\sum_{\lambda \in \Lambda} H_{\lambda}$ $=\left(H \cap G_{0}\right) \oplus \sum_{\lambda>0} H_{\lambda}$.

CoROllaRy 1. Let $G$ satisfy the conditions of Theorem 1 and $n G \leqq H$ $\leqq G$ for some $n \neq 0$. Then $H=\left(H \cap G_{0}\right) \oplus \sum_{\lambda>0} H_{\lambda}$, where $H_{\lambda} \cong G_{\lambda}$ for $\lambda>0$ and $n G_{0} \leqq H \cap G_{0} \leqq G_{0}$.

Received by the editors April 9, 1962 and, in revised form, January 23, 1963. 
This corollary follows immediately from the fact that $H^{a+1} \neq H^{a}$.

CoROLlaRY 2. Let $H$ be torsion free and $n H \leqq G \leqq H$ for some $n \neq 0$, where $G$ is as in Theorem 1. Then $H=\sum_{\lambda \in \Lambda} H_{\lambda}$ with $H_{\lambda} \cong G_{\lambda}$ for $\lambda>0$ and $n H_{0} \leqq G_{0} \leqq H_{0}$. Moreover, if $G_{0}$ is pure in $H, H_{0}$ can be taken to be $G_{0}$.

Proof. Since $n G \leqq n H \leqq G$, Corollary 1 gives $n H=\left(n H \cap G_{0}\right)$ $\oplus \sum_{\lambda>0} K_{\lambda}$ with $K_{\lambda} \cong G_{\lambda}$ for each $\lambda>0$. The map $x \rightarrow(1 / n) x$ is an isomorphism of $n H$ onto $H$ so that $H=(1 / n)\left(n H \cap G_{0}\right) \oplus \sum_{\lambda>0} H_{\lambda}$ with $H_{\lambda}=(1 / n) K_{\lambda} \cong G_{\lambda}$ for $\lambda>0$ and, taking $H_{0}=(1 / n)\left(n H \cap G_{0}\right)$, with $n H_{0} \leqq G_{0} \leqq H_{0}$. Finally if $G_{0}$ is pure in $H$, then $n H \cap G_{0}=n G_{0}$ so that $H_{0}=(1 / n)\left(n H \cap G_{0}\right)=G_{0}$. Corollaries 1 and 2 give a generalization of a theorem of R. A. Beaumont and R. S. Pierce [2, Theorem 9.5 and Corollary 9.6]. When $G_{0}$ is taken to be zero, Theorem 1 and its corollaries apply to completely decomposable groups.

CoRollaRy 3. Let $G$ be a torsion free group, $n G \leqq H \leqq G$ and $H$ is a completely decomposable homogeneous group (every element is of the same type). Then $G$ is isomorphic to $H$.

THEOREM 2. Let $G$ be a finite rank torsion free group with types in a chain. Then $G$ is completely decomposable if and only if for any regular subgroup $H$, with $r(H)$ (rank of $H)=r(G), G / H$ is finite.

Proof. Assume $G=\sum_{i=1}^{n} G_{i}, r\left(G_{i}\right)=1$ and $H$ is as stated in the theorem. Then by regularity, $H \cap G_{i}$ are of finite index in $G_{i}$. Since $H$ contains $\sum_{i=1}^{n}\left(H \cap G_{i}\right)$ which is of finite index in $G$, we see that the condition is necessary. In order to prove sufficiency, we choose a special set of $n$ independent elements $h_{1}, h_{2}, \cdots, h_{n}$ in the following manner. As $G$ is of finite rank and with types in a chain, there must be an element $h_{1} \neq 0$ with maximal type in $G$ otherwise we shall be led to the contradiction that $G$ is not of finite rank. Suppose we have selected $h_{1}, \cdots, h_{r}$ which are independent and $K_{r}$ to be the set of elements which are independent to $h_{1}, \cdots, h_{r}$. In $K_{r}$ we choose $h_{r+1}$ with maximal type among the elements in $K_{r}$. With this selection we have a maximal independent set $\left\{h_{1}, \cdots, h_{n}\right\}$ and $H=\sum_{i-1}^{n}\left\langle h_{i}\right\rangle_{*}\left(\langle x\rangle_{*}\right.$ is the pure subgroup generated by $x)$ is regular. For if $H$ is not regular, there exists $h=\sum_{i=1}^{m} x_{i}\left(x_{i} \in\left\langle h_{i}\right\rangle_{*}\right.$ and $\left.x_{m} \neq 0\right)$ such that $T_{G}\left(x_{m}\right)$ $=T_{H}(h)<T_{G}(h)$. Then if $h_{1}, \cdots, h_{m-1}, h$ is independent and $T(h)$ $>T\left(h_{m}\right)$ we get a contradiction about the choice of $h_{i}$. Hence $H=\sum_{i=1}^{n}\left\langle h_{i}\right\rangle_{*}$ is regular and $G / H$ finite. By Corollary 2, we have that $G$ is completely decomposable. When we make the sharper condition that $G$ is homogeneous in Theorem 2, we get a theorem of Baer $[1$, Theorem 48.1 , p. 173$]$. 


\section{REFERENCES}

1. L. Fuchs, Abelian groups, Publ. House of the Hungarian Academy of Sciences, Budapest, 1958.

2. R. A. Beaumont and R. S. Pierce, Torsion-free rings, Illinois J. Math. 5 (1961), 61-98.

National TaIwan University

\section{ISOMETRIC ISOMORPHISMS OF MEASURE ALGEBRAS}

B. E. JOHNSON

1. Introduction. We shall prove that two locally compact topological groups with isometrically isomorphic measure algebras are themselves isomorphic. This is similar to a result of Wendel (see [2]) proved for group algebras. We first characterize the absolutely continuous measures on a group in terms of the metric and algebra properties of the measure algebra. It then follows that if two locally compact groups have isometrically isomorphic measure algebras then they have isometrically isomorphic group algebras, and so our result follows from Wendel's theorem.

2. Singularity in terms of norm. Throughout this section $X$ will denote a locally compact Hausdorff space. The set $M(X)$ of bounded complex Radon measures on $X$ forms a Banach space (see [1, p. 57]).

LEMMA 1. If $\alpha, \beta$ are two complex numbers then

$$
|\alpha+\beta|+|\alpha-\beta| \leqq 2|\alpha|+2|\beta| \text {, }
$$

equality occurring if and only if $\alpha=0$ or $\beta=0$.

Proof. This result follows from the elementary inequalities $|\alpha \pm \beta| \leqq|\alpha|+|\beta|$ by considering the occasions in which these are both equalities.

THEOREM 1. Let $\mu_{1}, \mu_{2} \in M(X)$. Then $\mu_{1}$ and $\mu_{2}$ are mutually singular if and only if

$$
\left\|\mu_{1}+\mu_{2}\right\|+\left\|\mu_{1}-\mu_{2}\right\|=2\left\|\mu_{1}\right\|+2\left\|\mu_{2}\right\| .
$$

Proof. Put $\nu=\left|\mu_{1}\right|+\left|\mu_{2}\right|$. Both $\mu_{1}$ and $\mu_{2}$ are absolutely continuous with respect to $\nu$, and so there are $\nu$-integrable functions $f_{1}, f_{2}$ on $X$, such that $\mu_{i}(E)=\left(f_{i} . \nu\right)(E)=\int_{E} f_{i}(t) d \nu(t), i=1,2$, for each Borel set $E$ in $X$. We have

Received by the editors December 1, 1962. 